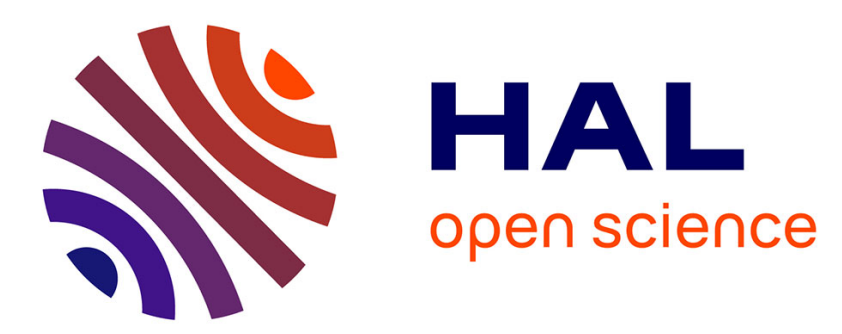

\title{
Channel flow LES with stochastic modeling of the sub-grid acceleration
}

\author{
Rémi Zamansky, Ivana Vinkovic, Mikhael Gorokhovski
}

\section{To cite this version:}

Rémi Zamansky, Ivana Vinkovic, Mikhael Gorokhovski. Channel flow LES with stochastic modeling of the sub-grid acceleration. 12th EUROMECH European Turbulence Conference, Sep 2009, Marburg, Germany. pp.637-640, 10.1007/978-3-642-03085-7_153 . hal-00683346

\section{HAL Id: hal-00683346 https://hal.science/hal-00683346}

Submitted on 28 Mar 2012

HAL is a multi-disciplinary open access archive for the deposit and dissemination of scientific research documents, whether they are published or not. The documents may come from teaching and research institutions in France or abroad, or from public or private research centers.
L'archive ouverte pluridisciplinaire $\mathbf{H A L}$, est destinée au dépôt et à la diffusion de documents scientifiques de niveau recherche, publiés ou non, émanant des établissements d'enseignement et de recherche français ou étrangers, des laboratoires publics ou privés. 


\title{
Channel flow LES with stochastic modeling of the sub-grid acceleration
}

\author{
R. Zamansky, I. Vinkovic and M. Gorokhovski
}

LMFA UMR 5509 CNRS Ecole Centrale de Lyon Université Claude Bernard Lyon 1, 36 avenue Guy de Collongue, 69134 Ecully Cedex, France

remi.zamansky@ec-lyon.fr, ivana.vinkovic@ec-lyon.fr, mikhael.gorokhovski@ec-lyon.fr

In [1] the large eddy simulation with stochastic modeling of the sub-grid acceleration (LES-SSAM) for homogeneous turbulence was proposed. The main motivation of this approach is to account for intermittency of the flow at subgrid scales, by emphasizing the role of sub-grid acceleration. In this paper, we develop further this approach in order to simulate a high Reynolds number channel flow. We proposed a new sub-grid acceleration model and compared it with DNS and standard LES.

In LES-SSAM approach of [1], the total acceleration is decomposed on filtered (resolved) and unresolved part, in a way that the instantaneous model velocity field $\hat{u}_{i}$ is resolved by:

$$
\hat{a}_{i}=\frac{\partial \hat{u}_{i}}{\partial t}+\hat{u}_{k} \frac{\partial \hat{u}_{i}}{\partial x_{k}}=-\frac{1}{\rho} \frac{\partial \hat{p}}{\partial x_{i}}+\frac{\partial}{\partial x_{k}}\left(\nu+\nu_{t}\right)\left(\frac{\partial \hat{u}_{i}}{\partial x_{k}}+\frac{\partial \hat{u}_{k}}{\partial x_{i}}\right)+\hat{a}_{i}^{\prime} ; \quad \frac{\partial \hat{u}_{k}}{\partial x_{k}}=0
$$

where $\nu$ is the viscosity, and $\nu_{\text {turb }}$ is given by the Smagorinsky model.

In the modeling of the non-resolved acceleration $\hat{a}_{i}^{\prime}$ for turbulent channel flow, our assumption is to emulate its modulus, $|a|$, by the product of the typical velocity increment, $u_{*}^{2} / \nu \Delta\left(u_{*}\right.$ is the friction velocity and $\Delta$ is the characteristic cell size) and the frequency, $f$, considered as stochastic variable:

$$
\hat{a}_{i}^{\prime}=|a|(t) e_{i}(t)=f u_{*}^{2} / \nu \Delta e_{i}
$$

Here $e_{i}$ is a random unit vector of orientation, also simulated in this paper. The frequency $f$ is supposed to be evolved with non-dimensional parameter $\tau=$ $-\ln \left(\frac{h-y}{h}\right)$, where $h$ represents the channel half-width, and $y$ is the distance from the wall. The stochastic equation is derived here in the framework of scaling symmetry [2], and has the following form:

$$
d f=\left[\langle\ln \alpha\rangle+\left\langle\ln ^{2} \alpha\right\rangle / 2\right] f d \tau+\sqrt{\left\langle\ln ^{2} \alpha\right\rangle / 2} f d W(\tau)
$$


$d W(\tau)$ is a Wiener process. The parameters of eq. 3 are $-\langle\ln \alpha\rangle=\left\langle\ln ^{2} \alpha\right\rangle=$ $R e_{+}^{1 / 3}$, where $R e_{+}=u_{*} h / \nu$. For the starting condition, $\tau=0$, in this stochastic process, we introduce the characteristic value of frequency prescribed on the wall $f_{+}=\lambda / u_{*}$, where $\lambda$ is determined, as Taylor scale, but in the framework of definitions of wall parameters. This is done as follows: the Reynolds number, based on friction velocity, is $R e_{+}=u_{*} h / \nu=h / y_{0} \approx R e_{h}^{3 / 4}$ where $y_{0}$ is the thickness of the viscous layer, and $R e_{h}$ is the Reynolds number based on the center-line velocity. One then yields: $\lambda \approx h R e_{h}^{-1 / 2} \approx h R e_{+}^{-2 / 3}$. Similar to Kolmogorov-Oboukhov 62 , the starting condition for the random path, given by eq. 3 , is sampled from the stationary log-normal distribution of $f / f_{+}$with parameters $\sigma^{2}=\ln 2$ and $\mu=-\frac{1}{2} \sigma^{2}$, such that $\langle f\rangle=\left(\left\langle f^{2}\right\rangle-\langle f\rangle^{2}\right)^{1 / 2}=f_{+}$. Hence this stochastic process for frequency will relax $f$ from a log-normal distribution on the wall $(\tau=0)$ to the power distribution with increasing the distance to the wall $(\tau \rightarrow \infty)$. The distributions of the frequency predicted by this stochastic process, on different wall-normal distances, are compared in fig. 1a with the evolution of the frequency computed from DNS, via eq. 2. It is seen that the model of frequency allows to predict the DNS relatively well.

In addition to the stochastic simulation of the unresolved modulus of acceleration, we simulate also the orientation vector $e_{i}$. This is done by a random walk evolving on the surface of a sphere of unity radius. First the computation of $e_{i}$ from DNS was performed. The result are shown in fig. 1b. It is seen that $e_{i}$ relaxes toward isotropy with increasing distance from the wall. In order to represent this tendency toward isotropy, we implement the Kubo oscillator with a real coefficient $\alpha$ for the random motion on the sphere. Each position increment of the random walk is given by $\zeta=\alpha d W(\tau)=\sqrt{\frac{\ln R e_{+}}{2}} W(\tau)$, and the direction $\beta$, at each time step, is chosen randomly from the uniform distribution. As $\tau$ increases, the random walk covers all the surface of the sphere. In fig. $1 \mathrm{~b}$ the simulated distribution of $\theta=\sin ^{-1}\left(e_{y}\right)\left(e_{y}\right.$ is the normal to the wall direction) is compared with unresolved acceleration from DNS. The last one was computed as difference between actual acceleration, given by DNS and its filtered value on the scale $\Delta$.

The a posteriori tests of this model is performed by comparing the LESSSAM with standard LES and DNS, for three Reynolds numbers, $R e_{+}=590$, $R e_{+}=1000$ and $R e_{+}=2000$. We used our own DNS data, as well as the DNS data from [3] and [4]. For LES and LES-SSAM simulations the classical Smagorinsky model with a wall damping function for the turbulent viscosity has been applied. The grid size is $64 \times 64 \times 64$, and its resolution is: $\Delta x^{+} \times\left(\Delta y_{\min }^{+} \sim \Delta y_{\max }^{+}\right) \times \Delta z^{+}=87 \times(0.71 \sim 29) \times 29,147 \times(1.2 \sim 49) \times 49$ and $295 \times(2.4 \sim 98) \times 98$ for $R e_{+}=590,1000$ and 2000 respectively. As shown on fig. 2, the profiles of mean velocity and of standard deviation of the velocity fluctuations, predicted by LES-SSAM approach, are improved notably. Fig. 3a illustrates the computation of turbulent and viscous stresses. It is seen that computation of momentum fluxes in the wall-normal direction, is 

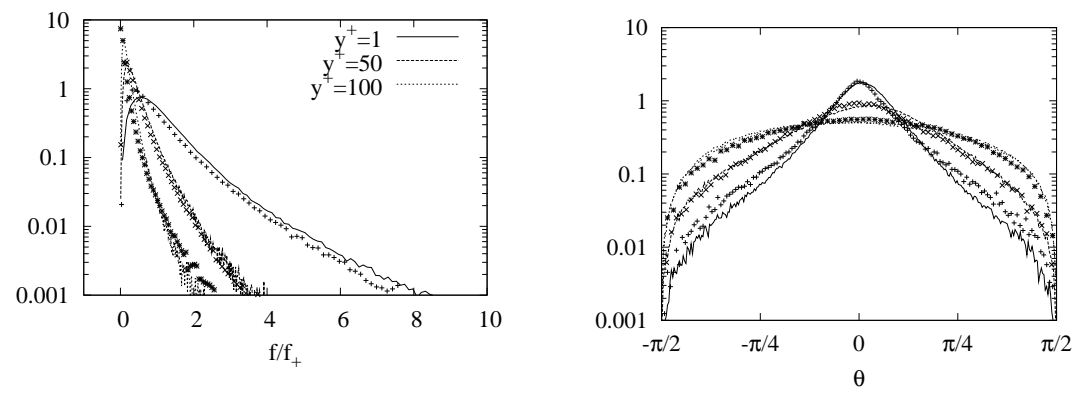

Fig. 1. (a): Distribution of $f / f_{+}$from SSAM (cross) and comparison with DNS (line) at $R e_{+}=590$, for several distances from the wall. (b): Distribution of $\theta=$ $\sin ^{-1}\left(e_{y}\right)$ for unresolved acceleration from DNS (line) and from SSAM (cross), for $R e_{+}=590$, and for several distances from the wall $\left(y^{+}=3, y+=10\right.$ and $\left.y^{+}=30\right)$.
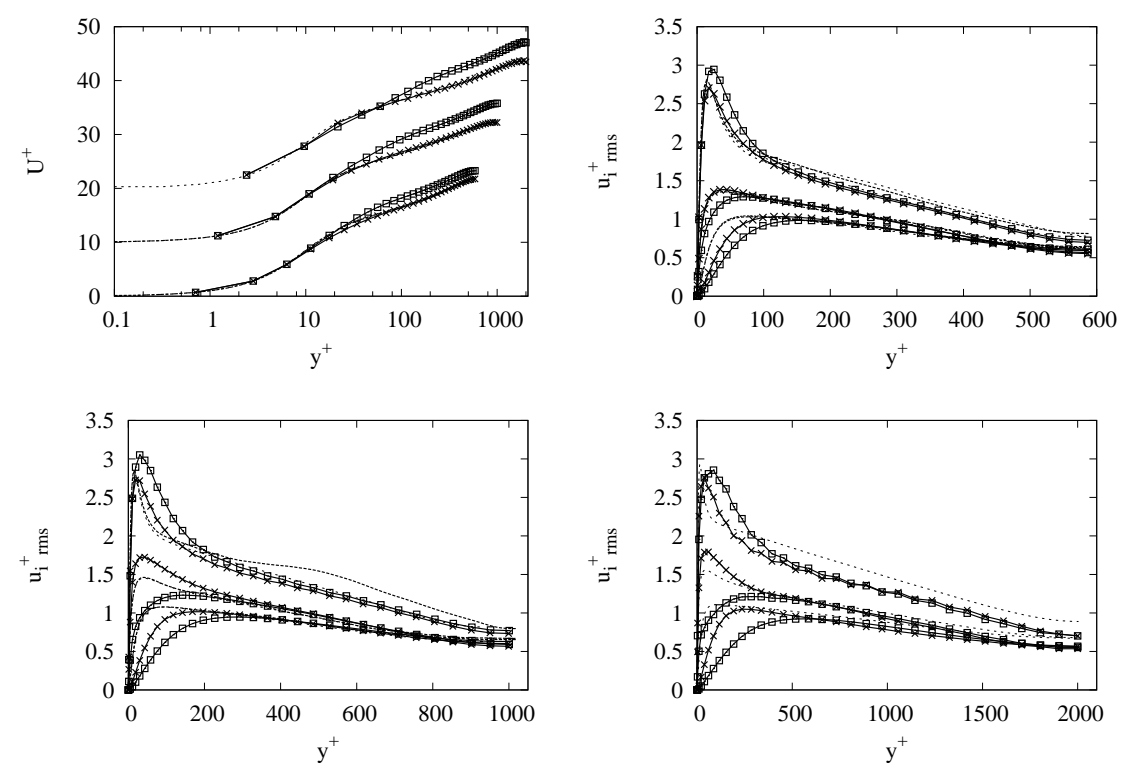

Fig. 2. (a): Streamwise mean velocity, for $R e_{+}=590, R e_{+}=1000$ and $R e_{+}=2000$ from bottom to top, respectively, shifted by 10 wall units upward. Standard deviation of streamwise $(u)$, spanwise $(w)$ and normal $(v)$ velocity (in wall unites), for (b): $R e_{+}=590,(\mathrm{c}): R e_{+}=1000$ and (d): $R e_{+}=2000$. Square: LES; cross: LES-SSAM; dash: DNS (only for $R e_{+}=590$ and $R e_{+}=1000$ ); dots: DNS from [3] for $R e_{+}=590$ and from [4] for $R e_{+}=1000$ and $R e_{+}=2000$. 
improved in comparison with LES. Velocity spectra are shown in fig. 3b. The excessive damping of energy on small scales, which is inherent to LES, is reduced by LES-SSAM. Fig. 3c represents the evolution of the longitudinal autocorrelation coefficient for the streamwise velocity component along the channel. Improvement of the decorrelation length can be seen as well, when LES-SSAM is used. Fig. 3d displays distribution of acceleration component in the spanwise direction. In agreement with the DNS, the LES-SSAM distribution exposes stretched tails, as a manifestation of the close to the wall intermittency, which is not the case by using LES.
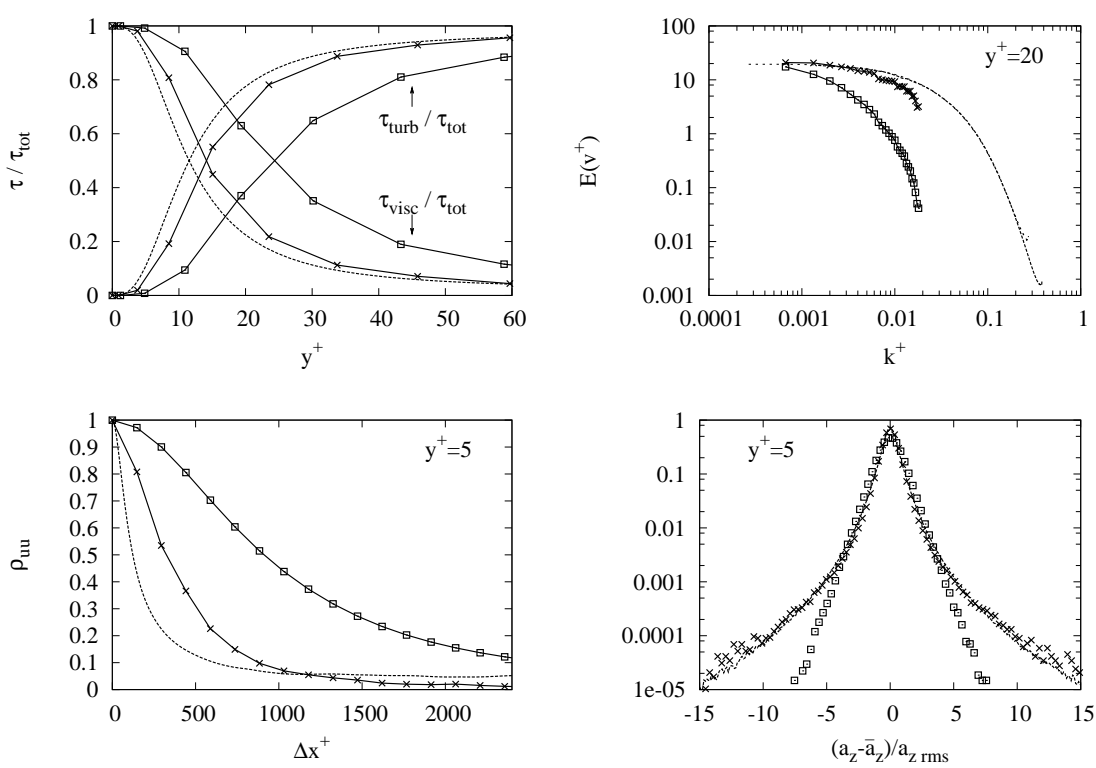

Fig. 3. Reynolds number of $R e_{+}=1000$. (a): Fractions of turbulent $\tau_{\text {turb }}=-\rho\left\langle u^{\prime} v^{\prime}\right\rangle$ and viscous $\tau_{v i s c}=-\rho \nu\left\langle\frac{\partial u}{\partial y}\right\rangle$ stresses compared to the total one $\tau_{\text {tot }}=\tau_{\text {visc }}+\tau_{\text {turb }}$. (b): Normalized longitudinal 1-D spectra of streamwise velocity for $y^{+}=20$. (c): Longitudinal autocorrelation of streamwise velocity at $y^{+}=5$. (d): Distribution of spanwise component of acceleration for $y^{+}=5$. Square: LES; cross: LES-SSAM; dash: DNS; dots: DNS from [4] (uniquely for (b)).

\section{References}

1. V. Sabelnikov, A. Chtab \& M. Gorokhovski, Advances in Turbulence XI, 11th EUROMECH European Turbulence Conference, 117 209-211 (2007).

2. M. Gorokhovski \& V. L. Saveliev, J. Phys. D: Appl. Phys. 41, 085405 (2008).

3. R. D. Moser, J. Kim \& N. M. Mansour, Phys. Fluids 11, 943-945 (1999).

4. S. Hoyas \& J. Jiménez, Phys. Fluids 20, 101511 (2008). 